\title{
Fusing Multilabel Deep Networks for Facial Action Unit Detection
}

\author{
Mina Bishay and Ioannis Patras \\ School of Electronic Engineering and Computer Science, Queen Mary University of London, London, UK
}

\begin{abstract}
The automatic detection of the activation of facial muscles, i.e. the detection of the so called facial Action Units (AUs), has received significant attention due to the application of facial expression analysis/recognition in areas such as affect recognition or behavior analysis. However, the recognition of subtle expressions is a challenging task that requires a multimodal approach where several sources of information are used. In this paper, we follow such an approach and propose a novel Deep Learning architecture that fuses information from several specialized Deep Neural Networks (DNNs) each of which models a different aspect of the problem in question. At the core of our approach is a novel dynamic adaptation of the Deep Network cost function so as to deal with the data imbalances that are inherent in multilabel classification problems - this allows crossdatabase training. We show the benefits of the proposed training approach and how different architectures are more suitable for particular AUs. Extensive experimental results show that our multi-modal approach outperform the state of the art by a considerable margin.
\end{abstract}

\section{INTRODUCTION}

Human affect, emotions, and personality can be identified, mainly from the facial information [2]. Automatic facial analysis has been an active research area in computer vision in the last twenty years. Ekman and Friesen put the cornerstone for studying facial expressions, by proposing the Facial Action Coding System (FACS) [7]. FACS has different combinations of facial muscle movements, that result in different facial expressions. These muscle movements are represented by Action Units (AUs). Manual annotation of these AUs is a very hard task as it requires hours for annotating a minute of a video. Subsequently, building an automatic and reliable AUs detection system will offer a powerful tool for different fields (e.g. Psychological field [6], [16]), that use manual annotation for studying facial behaviour. Moreover, it will have a great impact on the current challenging facial-based applications (e.g. affect recognition [30]).

Automatic AUs annotation has been the focus of many researchers for a long time. However, there is still a gap between the state-of-the-art results and the performance needed in several face-analysis applications. This is due to head pose variation, appearance differences, and limitations of the available datasets, i.e. lack of sufficient positive samples for certain AUs, as well as recording in controlled conditions.

Inspired by the remarkable success of Deep learning in several Computer Vision problems [11], [13], [15], [18], several works apply this paradigm for AUs detection [10], [32]. Literature review shows that learned features give better performance than the hand-crafted ones. However, many works address the problem of AUs detection as a binary classification problem, where a different model is built for 978-1-5090-4023-0/17/\$31.00 @2017 IEEE each $\mathrm{AU}$, and ignoring in this way informative correlations between the different AUs. Other works that pose the problem as a multilabel classification problem are faced with the inherent imbalance of the data, since the number of positive examples for different AUs vary wildly. Moreover, combining different datasets in the training process becomes infeasible, as they being annotated with different AUs.

In this paper, we propose a Deep Learning architecture that fuses information from several sources. The proposed architecture consists of eight Deep Networks, each one of which is specialized in different aspects of the problem, as depicted in fig. 1. At the first stage, two Convolutional Neural Networks (CNNs) are used for learning deep appearance features based on cropped face images, and two MultiLayer Perceptrons (MLPs) for learning distinctive geometric features based on facial landmarks locations. A multilabel classifier is used in each CNN and MLP to extract the framebased AUs correlations. At the second stage, a Recurrent Neural Network (RNN) is placed at the top of each of the four networks of the first stage so as to learn the spatiotemporal AUs correlations over consecutive video frames. At the third stage the predictions of those eight models are fused. Experimental results on well-known datasets show that the different networks perform significantly different in detecting diverse AUs and that the combined architecture performs considerably better than any single network.

In the heart of our architecture is a method for training each of the individual Deep Networks as a multilabel classifier that at test phase simultaneously detects all AUs. This is in contrast to other approaches that treat the problem as several binary classification problems [1], [10], [27], one for each $\mathrm{AU}$, and fail, therefore, to extract features that are shared between the different AUs or utilize patterns of co-occurrence. Here we make two contributions. First, we address a common issue in multilabel problems, and in particular in AU-annotated datasets that is the inherent data imbalance - while this can be solved efficiently in binary classification problems, for example with over/under sampling, in multilabel problems balancing the data (typically the current batch) with respect to one class (AU in our case) will inevitably result in unbalancing it with respect to another class. In this paper, we address this problem by proposing to adjust the cost term associated with each AU positive example with the ratio of negative to positive examples in the current batch and therefore control the back-propagated error.

The second contribution is that we address the problem of threshold selection at the output neurons at test time. In our architecture, in order to avoid threshold selection, each 


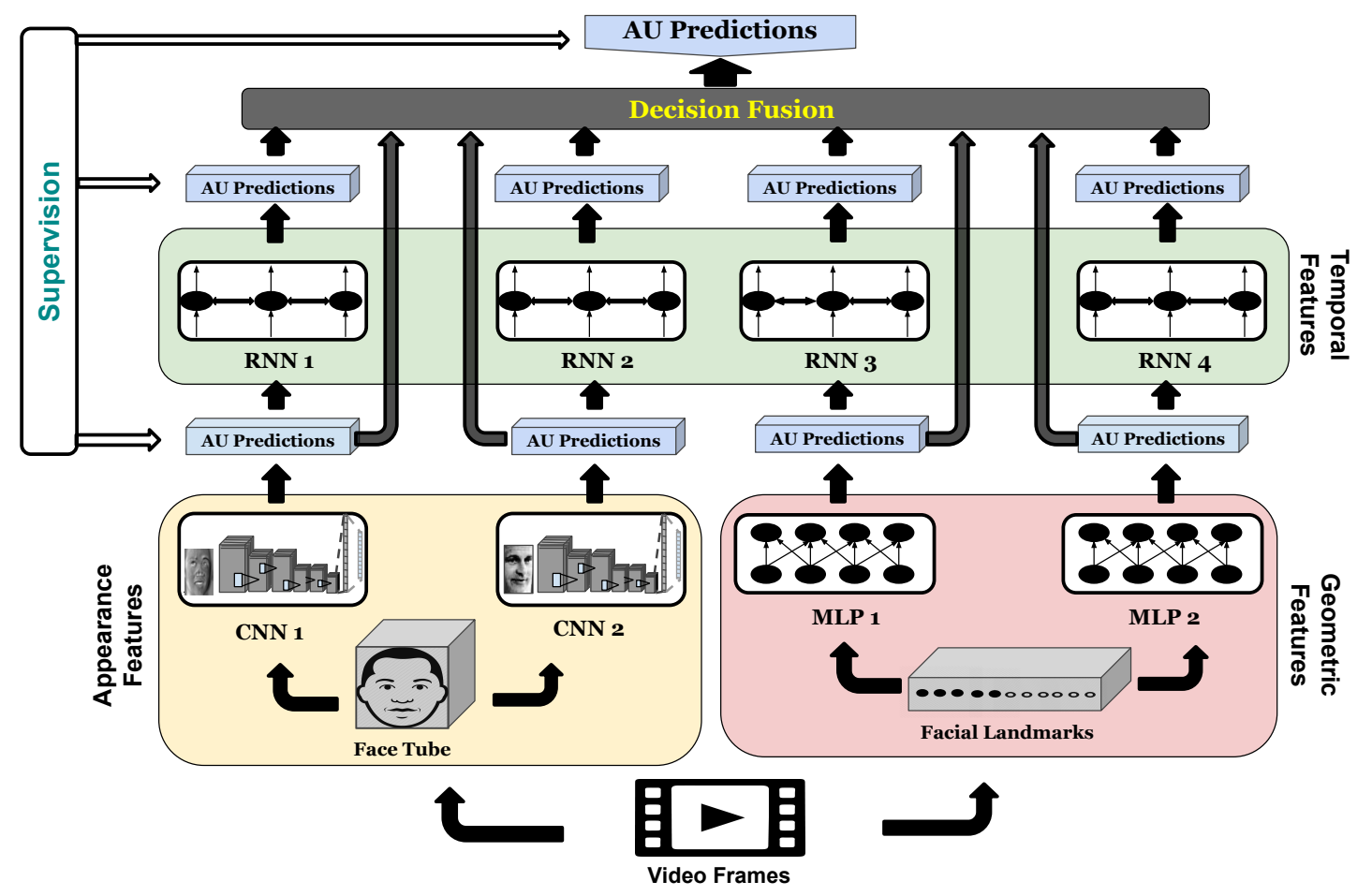

Fig. 1. The proposed architecture.

class is represented by 2 neurons, 1 for positive activation while other for negative activation. During training, those output neurons are supervised with complementary information, and during testing, the maximum of the 2 neurons is chosen to represent the activation. Those two contributions allow us to design a more general architecture that can be trained across several datasets and for the detection of many AUs. We have used in our analysis the four well-known spontaneous databases UNBC [21], DISFA [22], and FERA [27] (FERA includes two databases, BP4D and SEMAINE). The proposed architecture outperforms the state-of-the-art methods by a significant margin.

In section 2, we will review the related literature in AUs detection and highlight how the proposed method addresses their shortcomings. The proposed multilabel training scheme is described in section 3, and the proposed architecture with the fusion of several specialized Deep Networks is presented in section 4. Finally, experimental results and conclusion are given in section 5 , and section 6 , respectively.

\section{RELATED WORK}

Automatic AUs recognition has been the focus of many researchers for a long time. Different methods have been proposed, that differ in many aspects. In this section, we present some of the state-of-the-art methods, to illustrate the main trends and highlight their shortcomings.

One of the critical steps in AUs detection is the feature extraction. Extracted features can be divided roughly into hand-crafted [1], [14], [29], [31], and learned features [8], [9], [10], [32]. Each of these features can be further split into appearance and geometric ones. In [1], the hand-crafted appearance and geometric features are fused together for better performance. In spite of the great performance achieved by the learned features, the fusion of the deep appearance and geometric features have not been discovered yet in AUs detection. Moreover, Jung et al. proved in [12] that better accuracy can be achieved in emotion recognition when both of them are used. In this paper, both deep appearance and geometric features are fused to improve the feature extraction step.

The extracted features are then used for training several binary classifiers or a multilabel classifier. In [1], [10], [27], a binary classifier is used for each $\mathrm{AU}$, to learn AU-specific features. Using AU-specific classifier increases linearly the complexity, and the computational cost of the whole architecture. In [8], [9], a single multilabel classifier is used for different AUs, in order to learn general AU features, and the embedded spatial AUs correlations. In [32], a similar multilabel classifier is used, but replacing the conventional CNN general filters by a region-specific ones. The main drawback of this method is the large number of parameters (approx. 56 million), which easily make the network overfit when trained on limited data or subjects. Based on that, a single multilabel classifier is used in this paper to exploit the frame-based AUs correlations. Moreover, the multilabel classifier is modified to address the data imbalance and threshold selection problems.

Another aspect is the domain for extracting the features, that can either be spatial or spatio-temporal domain. Most of the existing works [8], [9], [32], focus on extracting features at the spatial domain. In [10], Jaiswal and Valstar proposed to 
extract the short-term spatio-temporal features by using a 3D $\mathrm{CNN}$, and the long-term ones by adding a bidirectional Long Short Term Memory (LSTM) to the 3D CNN. Although, the CNN-LSTM model shows a good performance in extracting spatio-temporal features, multiple single-label classifiers are trained, one for each AU, and therefore the AUs correlations are discarded. In the proposed architecture, a RNN is used on the top of a multilabel classifier to extract the spatio-temporal AUs correlations.

The normalization of the features or the face images using the subject's neutral face helps in extracting more discriminative features. In [8], Ghosh et al. proposed to normalize face images using the subject's mean (neutral) face. In [1], Baltrusaitis et al. proposed to normalize the extracted features by using features calculated from the subject's median (neutral) face. Although, subtracting the mean/median face can improve the performance significantly compared to using face images directly. The calculated mean/median face is not always the neutral face. Larger improvement can be achieved if an accurate neutral face image is fed to the network. In order to tackle the neutral face detection, two networks are used, one based on mean (neutral) face subtraction, while the other is based on original face images. The two networks complement each other for better AUs detection results.

Finally, different AU-annotated databases are available for the research community. The way that these databases are used for training and testing affects the AUs classification and reflects the generalization of the classifier. In [10], [29], models are trained and tested on the same database. In [8], Ghosh added a way of using one database for training, while other for testing. In [1], Baltrusaitis combined different databases for specific AU, in order to increase the number of training examples. Although combining databases can improve classifier performance by avoiding overfitting, it seems a hard task when a multilabel classifier is used since not all of the databases are annotated to the same AUs. In this paper, the proposed architecture is trained on all databases by back-propagating only the errors coming from the annotated AUs in each database.

\section{Multilabel training SCheme}

AUs detection can be naturally seen as a multilabel classification problem in which, at each example, one or typically more AUs are activated. Several works address AUs detection as independent binary classification problems, where a different classifier is built for each of the AUs. Then, a ranking or thresholding technique is used to give final predictions. However, the complexity of such an approach increases as the number of classes increase. Moreover, label dependencies which are very strong in the AUs detection problem, are discarded in this way, and the networks are likely to overfit on AUs that have a few annotated examples. In this paper, we follow [24] that extends a common singlelabel multiclass classifier to a multilabel multiclass classifier, in order to exploit embedded correlations and reduce complexity. Such multilabel classifiers are employed in each of our eight specialized models that we will describe in the next section.

Deep learning techniques have many parameters that can easily overfit when a limited number of subjects, or data are used in training. In order to avoid overfitting and extract distinctive AU-related features, different databases should be used in the training process. The main impediment for using combined databases in training a multilabel classifier is the unequal number of AUs annotated in these databases. In order to solve this problem and exploit all the available datasets, each image in the used databases is annotated in terms of 18 AUs, with a ground truth label $q \in\{0,1, N L\}$. The AU presence is labelled by $1, \mathrm{AU}$ absence by 0 , and $N L$ if the image is not annotated for this AU. The computed cost for the $N L$-labelled AUs is discarded, and does not take part in the average back-propagated cost. Therefore, the computed cost is only for the annotated AUs in each batch.

The first contribution that we make in this field is addressing the problem of threshold selection for AUs classification. Typically, in order to make a binary decision on whether the $\mathrm{AU}$ in question is activated or not, the corresponding neuron output is thresholded either at 0.5 or, more commonly, by a threshold that is chosen based on the training set. However, different conditions (e.g. head motion, lighting effects) can affect the neuron output, and therefore using a certain threshold for all images is not the best choice. In order to overcome this problem and choose the threshold automatically, each AU $i$ is represented by 2 neurons, one representing $\mathrm{AU}$ presence $A U_{1}^{i}$ while the other representing $\mathrm{AU}$ absence $A U_{0}^{i}$. During training they are supervised by complementary information, and during testing the one with the highest output is selected. Doing so allows the network to choose the threshold automatically according to the given input conditions - for example blurring or a darker image will affect both neurons in the same manner.

The second contribution that we make in this field is a scheme that addresses the problem of data imbalance. Data imbalance is a common problem in many applications including AUs detection and results in biasing the classifier towards the class with the most samples. Typically, positive examples are limited - this can be tackled by duplicating the positive examples (named "Oversampling"), or removing some negative examples (named "Undersampling") [4]; however, this is only possible in a binary classification problem - in a multilabel classification problem balancing the data with respect to one AU will result in imbalance with respect to other AUs. In this paper, we propose a new method for balancing the data in a multilabel classifier. For each batch in the training set, let us denote by $p_{i}$ the number of the positive examples and $n_{i}$ the number of negative examples for the AU $i$. Then, the ratio $r_{i}$ of the negative to positive examples is computed as:

$$
r_{i}= \begin{cases}\frac{n_{i}}{p_{i}}, & \text { if } n_{i} \text { and } p_{i} \neq 0 \\ 1, & \text { otherwise, }\end{cases}
$$

where the index $i \in\{1,2,3, \ldots, 2 K\}$ where $2 K$ is twice 
the number of AUs, as we use 2 neurons for each AU. Then, we create a weight matrix $M$, having the same size of the output batch. In the weight matrix, we set the 0-labeled examples by 1 , NL-labeled by 0 , and 1-labeled by $r_{i}$, where $r_{i}$ is given by eq. 1 , and is different for each $\mathrm{AU} i$. This weight matrix is multiplied elementwise by the output cost matrix. By doing so we adjust the misclassification cost of positive examples so as to prevent the biasing of the network towards the negative class when a few positive examples are available. We use the binary cross-entropy as a cost function. That is, the total batch cost is:

$$
\begin{gathered}
C=-\frac{1}{2 K} \sum_{i=1}^{2 K} \frac{1}{z_{i}} \sum_{j=1}^{b s} M_{i j}\left(t_{i j} \log q_{i j}+\left(1-t_{i j}\right) \log \left(1-q_{i j}\right)\right) \\
z_{i}=\sum_{j=1}^{b s} M_{i j}
\end{gathered}
$$

where $z_{i}$ denotes the sum of the weights at $\mathrm{AU} i$, bs the batch size, $t$ the target value and $q$ is the predicted value. Across the different databases, the multilabel classifier will learn general and discriminative features for AUs detection.

\section{Fusion ARChitecture}

Our architecture consists of multiple deep networks for facial AUs detection. Two CNNs are used for extracting appearance features, and two MLPs are used for extracting geometric features. Then, a RNN is built on the top of each of the spatial models to learn the spatio-temporal AUs correlations. Finally, the eight models' predictions are fused in a linear layer, in order to pick the best weights for each $\mathrm{AU}$, over the different models.

\section{A. Preprocessing Step}

Given any of the database spontaneous videos, preprocessing is a crucial step to ensure that a stream of aligned face images and landmarks are fed to our architecture. Face detection is an effective step, as all of the processing steps depend on it. Therefore, two face detectors were employed; OpenCV face detector, trained on frontal and profile faces, and Zhu-Ramanan face detector [33]. First, OpenCV is used for its robust and fast performance. Then, Zhu-Ramanan detector is used as a complementary model to process the failed frames, as OpenCV fails sometimes when the roll angle of the head pose increases. The extracted face images are passed for landmarks localization. The Supervised Descent Method [28] is used for extracting 49 landmarks. The detected landmarks are then aligned to a reference frame using Procrustes transform. The points that are invariant to facial expressions, like eye corners and nose tip are used for the alignment. Finally, the faces are cropped to $48 \times 48$, and converted to gray scale, and used with the landmarks locations as inputs to the CNNs and MLPs, respectively.

\section{B. Convolutional Neural Networks}

Following the great success achieved by AlexNet in image classification [15], CNNs have been extensively used for

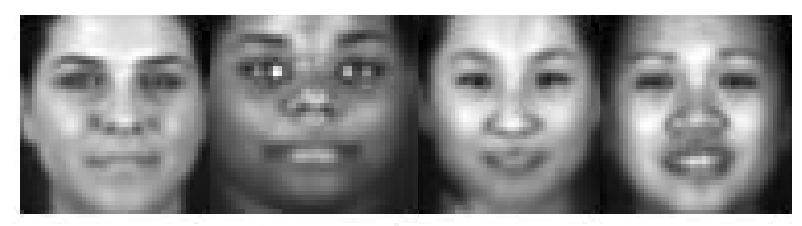

Fig. 2. Samples of subjects' mean face selected from BP4D dataset.

different Computer Vision problems in the last years. CNNs learn better appearance features than the designed ones. In this work, two CNNs are employed for extracting deep appearance features. The first CNN (called CNN1) is based on subtracting subject's mean face, which can learn meaningful features away from the appearance differences. The other CNN (called CNN2) is based on the original face images, this network can better learn AUs when the calculated neutral face is not accurate enough. More specifically, AUs are subtle and differ according to face appearance, shape, and dynamics, so subtracting the mean (neutral) face can avoid these differences by highlighting the AUs locations when its activated. The assumption of using the mean [8], or the median [1] of all subject's frames as the neutral face is not always accurate. Fig. 2 shows several subjects' mean faces, the first two are almost neutral, but in the last two the mouth is slightly opened (AU25). Subsequently, CNN1 works better for almost all AUs, but CNN2 is employed to better classify the mistaken AUs (e.g. AU25), due to the neutral face inaccuracy. CNN1 and CNN2 complement each other for better performance.

The CNNs take batches of $48 \times 48$ face images as input and randomly crop each into $44 \times 44$ smaller sub-image. Subimages are randomly flipped horizontally with a probability of 0.5 , in order to augment the data and avoid overfitting problems. At test time classification is done using 44x44 subimages cropped from the center of the original face images. Each CNN consists of 3 convolutional layers and 1 fully connected layer, each convolutional layer is followed by a maxpooling layer [19]. For the first and second convolutional layers, 64 filters of size 9x9 and 5x5 respectively are used, and for the last convolutional layer, 128 filters of size $5 \times 5$ are used. The activation function used in the convolutional layers is the rectified linear unit (ReLU) function [23]. Dropout is used for regularization to avoid overfitting [26]. Max-pooling layers have filters of size $2 \times 2$ in order to make the network translation invariant. The last layer consists of $K$ sigmoid units, where $K$ is equal to the number of the detected AUs. The CNNs are trained using stochastic gradient descent, with the same learning parameters: 0.005 learning rate, 0.9 momentum, and 0.25 dropout. The learning rate decays with increasing epochs at a rate of 0.001 for CNN1 and 0.0005 for CNN2.

\section{MultiLayer Perceptron}

The inspiration of using MLP along with CNN comes from its success in emotion recognition in [12]. The normalized 
landmarks' locations are used as input to the MLP. The main idea of using landmarks for AUs detection is that some AUs (e.g. AU25, AU26) are characterized by a large shift in their locations, that make the network easily learn better features for AUs detection. Our algorithm contains two MLPs trained on the extracted landmarks' positions. The first MLP (called MLP1) is trained using the 49 landmarks, normalized by subtracting subject's mean landmarks. The other MLP (called MLP2) is trained using the original 49 landmarks, normalized according to the method in [12]. The idea of using two MLPs is the same as with the CNNs, they complement each other when the subject's neutral landmarks are not accurately detected.

Each landmark location has two coordinates (x,y), so the length of the input feature vector is $49 \times 2=98$. Two hidden layers are used, each consisting of 600 neurons. The output layer consists of $K$ sigmoid units, where $K$ is the number of detected AUs. ReLU is used as the activation function after each hidden layer. Both MLPs are trained using stochastic gradient descent, and share the same learning parameters: 0.005 learning rate, 0.9 momentum, and 0.25 dropout.

\section{Recurrent Neural Networks}

Facial AUs are strongly correlated both in the spatial and temporal domains. Spatial AUs correlations are extracted by the multilabel classifier used in each of the MLPs and CNNs. In order to extract the temporal correlations, a Bi-directional Recurrent Neural Network (B-RNN) is employed [25]. The B-RNN transform a number of inputs $(X)$ to a number of outputs $(Y)$ based on the input values, and previous and future information. In [17], Hinton compared several RNNs over different tasks, and found that a simple RNN with ReLU and scaled identity initialization can give better performance compared to others like LSTM. Based on that, this RNN is used in our analysis. Outputs of the CNNs and MLPs are fed to the RNNs as inputs over different video frames. At frame $t$, the output $y_{t}$ is calculated as follows:

$$
\begin{gathered}
y_{t}=a\left(W_{\text {out }}^{f} h_{t}^{f}+W_{\text {out }}^{b} h_{t}^{b}+b_{\text {out }}\right), \quad t \in\{1,2, \ldots, T\} \\
h_{t}^{f}=a\left(W_{i n}^{f} x_{t}+W_{h}^{f} h_{t-1}^{f}+b_{h}^{f}\right) \\
h_{t}^{b}=a\left(W_{i n}^{b} x_{t}+W_{h}^{b} h_{t+1}^{b}+b_{h}^{b}\right)
\end{gathered}
$$

where $W_{\text {out }}^{f}$, and $W_{\text {out }}^{b}$ are the output weight matrices connecting the forward and backward hidden states to the output layer, respectively. $W_{i n}^{f}$, and $W_{i n}^{b}$ are the input weight matrices connecting the input layer to the forward and backward hidden states, respectively. $W_{h}^{f}$, and $W_{h}^{b}$ are the forward and backward hidden weight matrices, respectively. $b_{\text {out }}, b_{h}^{f}$, and $b_{h}^{b}$ are the output, forward and backward hidden bias vectors, respectively. $T$ is the length of video sequence. The activation function $a$ is the sigmoid function for the output layer, and ReLU function for the hidden layers.

The B-RNNs are trained on databases with different video lengths. In our analysis, videos are partitioned into segments of length 90 frames. The hidden layers weights are initialized by a scaled identity matrix, where 0.1 is chosen as the scale value. All the B-RNNs were trained by stochastic gradient descent, with a learning rate of 0.01 , gradient clipping at 1.0 , and batches of size 32 sequences.

\section{E. Decision Fusion Stage}

Appearance (CNNs) and geometric (MLPs) models have varying AUs detection performances. In order to exploit both models information/features, and tackle the defects in neutral face detection, decision fusion is employed for the eight deep models. Indeed, decision fusion will also fuse the spatial AUs correlations extracted by CNNs and MLPs in the multilabel classifiers, with the temporal ones extracted by the B-RNNs.

A linear model whose parameters are optimized with random search [3], [13], is used to combine the predictions from the different modality classifiers. In random search, one weight is given for each AU/class in each model, and the final AU prediction is the weighted sum of all models predictions. Random sampling of uniform distribution is used to get weights between 0 and 1 . Then, each class weights are normalized to 1 . The best sampled weights are chosen based on the best F1-score. In our architecture, 25,000 iterations are used initially, then a local random search is performed around the best weights chosen over different classes. The weights for the local search are sampled from a Gaussian distribution with a mean equal to the best chosen weights, and standard deviation std of 0.5 . The local search is repeated around the best chosen weights after every 1000 iterations, and at each time the $s t d$ is decreased by a factor of 0.8 and stopped when it is smaller than 0.001 .

\section{EXPERIMENTS}

Experimental setup. Four spontaneous databases are used in our experiments: UNBC, DISFA, and FERA (which includes two datasets, SEMAINE and BP4D). FERA is divided into training, validation, and testing sets. In our first experiment, the BP4D is used to show the effect of the proposed methods for data balancing and automatic threshold selection in a multilabel classifier. In the second experiment, UNBC, DISFA, and FERA training set are randomly combined for training our architecture. Then, the FERA validation set is used for testing in terms of 18 AUs, to show the AUs detection performance over the 8 deep models, as well as their fusion model. In the third experiment, the code of the trained model is submitted to the FERA organizers, in order to be tested on the FERA 2015 challenge. FERA challenge specifies 6 AUs on SEMAINE, and 11 AUs on BP4D, for challenging. Using the FERA platform, allows all participants to test their methods in similar conditions, for a better and fair evaluation. In the last experiment, 3-fold partitioning is implemented for the BP4D database, in order to compare the proposed method performance to the results reported in [5], [20], [31], [32].

Performance metrics. In this paper, accuracy and F1score are used for evaluating the performance of the proposed algorithm. Accuracy is a widely used and powerful metric, but when the ratio of the negative to positive examples is large, the detection accuracy of the positive class is almost neglected. On the other hand, F1-score depends mainly on 
TABLE I

F1-SCORE AND ACCURACY AT DIFFERENT STAGES OF THE PROPOSED MULTILABEL CLASSIFIER.

\begin{tabular}{|c|c|c|c|c|c|c|c|c|c|c|}
\hline AU & \multicolumn{2}{|c|}{ MLC } & \multicolumn{2}{c|}{ ATS } & \multicolumn{2}{c|}{ DB } & \multicolumn{2}{c|}{ ATS + DB } & \multicolumn{2}{c|}{$\begin{array}{c}\text { ATS + DB } \\
\text { + CDT }\end{array}$} \\
\hline & F1 & Acc & F1 & Acc & F1 & Acc & F1 & Acc & F1 & Acc \\
\hline AU1 & 0.514 & 83.02 & 0.517 & 84.84 & 0.502 & 83.73 & 0.551 & 81.13 & 0.502 & 73.73 \\
\hline AU2 & 0.356 & 78.27 & 0.358 & 82.20 & 0.357 & 81.81 & 0.371 & 76.82 & 0.403 & 73.73 \\
\hline AU4 & 0.545 & 80.23 & 0.510 & 81.14 & 0.555 & 81.67 & 0.556 & 78.74 & 0.515 & 74.28 \\
\hline AU6 & 0.775 & 77.30 & 0.787 & 79.10 & 0.792 & 78.19 & 0.791 & 78.65 & 0.805 & 77.86 \\
\hline AU7 & 0.735 & 68.75 & 0.736 & 70.04 & 0.745 & 69.46 & 0.743 & 70.70 & 0.763 & 69.12 \\
\hline AU9 & 0.269 & 92.00 & 0.229 & 93.04 & 0.269 & 92.69 & 0.351 & 89.84 & 0.349 & 83.49 \\
\hline AU10 & 0.804 & 75.40 & 0.836 & 79.03 & 0.816 & 76.70 & 0.809 & 76.50 & 0.846 & 79.50 \\
\hline AU12 & 0.861 & 83.23 & 0.859 & 82.98 & 0.856 & 82.19 & 0.865 & 83.79 & 0.868 & 83.71 \\
\hline AU14 & 0.647 & 64.88 & 0.613 & 63.92 & 0.685 & 66.31 & 0.628 & 62.80 & 0.648 & 61.98 \\
\hline AU15 & 0.371 & 78.15 & 0.287 & 79.78 & 0.345 & 76.67 & 0.454 & 76.17 & 0.465 & 69.87 \\
\hline AU17 & 0.616 & 71.34 & 0.596 & 74.40 & 0.629 & 71.97 & 0.637 & 73.15 & 0.656 & 70.42 \\
\hline AU23 & 0.398 & 79.54 & 0.352 & 82.26 & 0.423 & 79.49 & 0.465 & 75.82 & 0.461 & 73.42 \\
\hline AU24 & 0.445 & 83.82 & 0.287 & 82.55 & 0.397 & 81.83 & 0.525 & 83.29 & 0.562 & 82.49 \\
\hline AU28 & 0.363 & 95.33 & 0.429 & 96.70 & 0.426 & 96.46 & 0.416 & 95.17 & 0.403 & 94.89 \\
\hline Avg & 0.550 & 79.38 & 0.533 & 80.86 & 0.557 & 79.94 & 0.583 & 78.75 & 0.589 & 76.32 \\
\hline
\end{tabular}

the detection performance of the positive class, but the number of the true negatives does not take a part in the computation. In what follows we report both metrics.

Results. In the first experiment, we show the effect of adding Automatic Threshold Selection (ATS), Data Balancing (DB), as well as Cross-Dataset Training (CDT) to the MultiLabel Classifier (MLC). As an illustrative case we show the performance of one of the eight models, that is CNN1, on the BP4D dataset using a 2:1 ratio of training and validation. Results on 14 out of 18 AUs that are annotated in BP4D are reported. We report results for various combinations of ATS, $\mathrm{DB}$, and CDT. When ATS is not used, the AU threshold is chosen based on the best F1-score and when CDT is selected, several datasets, i.e. UNBC, DISFA, SEMAINE and BP4D are used for training.

Table I summarizes the obtained results for the different settings. Using only ATS seems to reduce the average F1score compared to the simple MLC - a reason for that is the increase in false negative for AUs in which the positive/negative ratio is very low, where one output neuron is biased towards the more frequent class (i.e. 0) and the other output neuron is biased towards the most infrequent class (i.e. 1). Our cost adaptation method for data balancing improves the MLC performance by $0.7 \%$ in F1-score, but larger improvement is obtained when adding the ATS with DB - the F1-score is improved by approx 3.3\%. Finally, using ATS and DB with the expansion of the training set adds an additional $0.6 \%$ to the F1-score, as better features are extracted. Based on that, ATS, DB, and CDT is used in the training of each of our 8 deep models.

In order to show the importance of fusing information from several sources, and how the different models perform on different AUs, the SEMAINE and BP4D validation sets are combined and used for testing our architecture. The F1score and accuracy over the different stages of the architecture are shown in Table II. By comparing the performance of the appearance (CNNs) and geometric (MLPs) features, we found that on average, the appearance features perform better. However, AUs performance varies over the MLPs and CNNs - typically, CNNs detect better AUs that are characterized by subtle change in the appearance (e.g. AU2, AU6, AU10, AU17), while MLPs perform better for AUs characterized by large displacement in landmarks locations (e.g. AU25, AU26, AU28).

The effectiveness of the neutral face subtraction can be inferred from the good performance achieved by CNN1 in comparison to $\mathrm{CNN} 2$. On average, CNN1 outperforms CNN2 on both F1-score and accuracy. CNN1 works better for most of the AUs, except some of those related to the mouth area (e.g. AU15, AU17, AU24, AU25). That is the result of the inaccuracy in neutral face estimation at the mouth region. In the same way, comparing the performance of MLP1 and MLP2 gives similar conclusions. In order to address the problem of the neutral face estimation and utilize both the appearance and geometric features, decision fusion is implemented. Adding the fusion step to the four spatial models leads to the second best performing model, where the F1-score improves by approximately 3\% and accuracy by $1 \%$ compared to the best spatial model CNN1.

The RNNs extract the spatio-temporal AUs correlations over different video frames. Table II, shows the effect of adding the RNN for each spatial model. The F1-score is not affected for CNN2 and MLP2, but for CNN1 and MLP1, the improvement gained is $1.5 \%$ and $2.5 \%$, respectively. Adding the RNN to the CNN1 led to the third best model. The decision fusion of the 4 spatial models with the 4 temporal models led to the best performing model, where the F1score improves by $0.28 \%$ and accuracy by $1.2 \%$ compared to the second best model. Fusion of the 8 models exploits the appearance and geometric features, spatio-temporal AUs correlations, and addresses the inaccuracy in neutral face detection, which helps in boosting the performance of the AUs detection.

The proposed architecture has been also tested on the FERA challenge, including the BP4D and SEMAINE testing sets. Table III and table IV show the obtained results on the BP4D and SEMAINE, respectively, with other results reported in the literature [1], [9], [10], [27], [29]. We achieved the best F1-score on BP4D dataset and the second best on the SEMAINE dataset. 
TABLE II

F1-SCORE AND ACCURACY OVER DIFFERENT MODALITIES IN THE PROPOSED ARCHITECTURE.

\begin{tabular}{|c|c|c|c|c|c|c|c|c|c|c|c|c|c|c|c|c|c|c|c|c|}
\hline \multirow[t]{2}{*}{$\mathbf{A U}$} & \multicolumn{2}{|c|}{ CNN1 } & \multicolumn{2}{|c|}{ CNN2 } & \multicolumn{2}{|c|}{ MLP1 } & \multicolumn{2}{|c|}{ MLP2 } & \multicolumn{2}{|c|}{$\begin{array}{l}\text { CNN1 } \\
\text {-RNN }\end{array}$} & \multicolumn{2}{|c|}{$\begin{array}{l}\text { CNN2 } \\
\text {-RNN }\end{array}$} & \multicolumn{2}{|c|}{$\begin{array}{l}\text { MLP1 } \\
\text {-RNN }\end{array}$} & \multicolumn{2}{|c|}{$\begin{array}{l}\text { MLP2 } \\
\text {-RNN }\end{array}$} & \multicolumn{2}{|c|}{$\begin{array}{l}\text { CNN-MLP } \\
\text { Fusion }\end{array}$} & \multicolumn{2}{|c|}{$\begin{array}{c}\text { CNN-MLP } \\
\text {-RNN Fusion }\end{array}$} \\
\hline & F1 & Acc & F1 & Acc & F1 & Acc & F1 & Acc & F1 & Acc & F1 & Acc & F1 & Acc & F1 & Acc & F1 & Acc & F1 & Acc \\
\hline AU1 & 0.469 & 80.59 & 0.261 & 67.38 & 0.466 & 79.68 & 0.323 & 68.74 & 0.489 & 82.80 & 0.263 & 64.82 & 0.458 & 76.50 & 0.308 & 55.88 & 0.453 & 80.43 & 0.466 & 81.94 \\
\hline AU2 & 0.448 & 80.31 & .264 & 67.30 & 0.391 & 77.92 & 0.274 & 68.42 & 0.432 & 82.06 & 0.273 & 65.32 & 0.407 & 78.28 & 0.298 & 60.94 & 0.441 & 81.86 & 0.439 & 82.40 \\
\hline AU4 & 0.508 & 86.44 & 0.399 & 79.90 & 0.438 & 81.82 & 0.390 & 80.48 & 0.508 & 87.38 & 0.392 & 80.51 & 0.431 & 76.61 & 0.296 & 61.27 & 0.558 & 87.89 & 0.552 & 88.52 \\
\hline AU6 & 0.750 & 83.55 & 0.703 & 78.24 & 0.688 & 80.01 & 0.681 & 75.90 & 0.769 & 84.44 & 0.724 & 80.78 & 0.702 & 78.97 & 0.652 & 70.42 & 0.772 & 84.80 & 0.775 & 85.17 \\
\hline AU7 & 0.703 & 78.51 & 0.696 & 76.06 & 0.660 & 75.62 & 0.676 & 74.17 & 0.718 & 78.91 & 0.693 & 77.25 & 0.670 & 71.88 & 0.620 & 60.92 & 0.718 & 79.20 & 0.718 & 79.38 \\
\hline AU9 & 0.237 & 91.73 & 185 & 90.18 & 0.204 & 91.86 & 0.122 & 90.35 & 0.241 & 90.04 & 0.179 & 93.6 & 0.169 & 84.20 & 0.09 & 70.10 & 0.255 & 91.17 & 0.288 & 93.28 \\
\hline AU10 & 0.805 & 82.28 & .761 & 78.26 & 0.707 & 74.16 & 0.760 & 76.22 & 0.815 & 82.77 & 0.767 & 78.25 & 0.750 & 74.19 & 0.750 & 71.94 & 0.803 & 81.96 & 0.803 & 82.01 \\
\hline AU12 & 0.828 & 82.06 & 0.819 & 79.67 & 0.796 & 79.17 & 0.821 & 80.65 & 0.833 & 82.16 & 0.817 & 79.39 & 0.807 & 80.11 & 0.826 & 81.11 & 0.841 & 83.16 & 0.841 & 83.17 \\
\hline AU15 & 0.356 & 76.02 & .373 & 74.54 & 0.329 & 76.41 & 0.283 & 68.03 & 0.378 & 79.77 & 0.369 & 77.17 & 0.342 & 71.52 & 0.281 & 56.54 & 0.398 & 78.74 & 0.409 & 80.98 \\
\hline AU17 & 0.573 & 73.11 & 0.584 & 70.71 & 0.531 & 68.97 & 0.516 & 60.46 & 0.582 & 74.63 & 0.585 & 70.90 & 0.563 & 68.05 & 0.544 & 59.77 & 0.602 & 74.09 & 0.603 & 74.97 \\
\hline AU23 & 0.398 & 83.07 & 0.383 & 78.75 & 0.379 & 81.75 & 0.337 & 75.97 & 0.395 & 80.85 & 0.385 & 81.43 & 0.350 & 77.70 & 0.294 & 65.47 & 0.419 & 82.40 & 0.427 & 84.00 \\
\hline AU24 & 0.403 & 84.30 & 0.444 & 84.39 & 0.403 & 82.47 & 0.330 & 75.29 & 0.4 & 85.94 & 0.458 & 84.82 & 0.432 & 80.11 & 0.349 & 69.43 & 0.417 & 85.08 & 0.430 & 85.99 \\
\hline AU25 & 0.675 & 74.34 & 0.735 & 76.86 & 0.688 & 77.65 & 0.746 & 74.36 & 0.672 & 73.24 & 0.739 & 77.20 & 0.772 & 80.69 & 0.780 & 78.12 & 0.766 & 80.81 & 0.775 & 81.23 \\
\hline AU26 & 0.373 & 78.43 & 0.319 & 80.76 & 0.373 & 79.65 & 0.424 & 70.16 & 0.457 & 81.18 & 0.327 & 79.45 & 0.477 & 74.61 & 0.420 & 61.29 & 0.344 & 81.75 & 0.355 & 81.99 \\
\hline AU28 & 0.379 & 96.32 & 0.206 & 89.94 & 0.384 & 95.62 & 0.250 & 92.18 & 0.393 & 95.91 & 0.186 & 86.37 & 0.416 & 94.96 & 0.277 & 89.76 & 0.509 & 97.00 & 0.486 & 96.81 \\
\hline AU43 & 0.291 & 92.46 & 0.205 & 87.34 & 0.179 & 92.88 & 0.269 & 89.28 & 0.289 & 91.79 & 0.169 & 87.19 & 0.290 & 87.15 & 0.219 & 63.77 & 0.353 & 93.40 & 0.340 & 93.83 \\
\hline AU45 & 0.369 & 69.79 & 0.288 & 69.63 & 0.355 & 68.13 & 0.325 & 59.75 & 0.375 & 70.90 & 0.278 & 65.73 & 0.366 & 69.40 & 0.331 & 62.66 & 0.394 & 72.08 & 0.398 & 72.36 \\
\hline Avg & 0.506 & 81.38 & 0.455 & 77.64 & 0.472 & 79.51 & 0.450 & 74.87 & 0.520 & 82.02 & 0.454 & 77.57 & 0.497 & 77.31 & 0.448 & 66.17 & 0.536 & 82.67 & 0.539 & 83.87 \\
\hline
\end{tabular}

TABLE III

F1-SCORE ON THE BP4D TESTING SET.

\begin{tabular}{|c|c|c|c|c|c|c|c|}
\hline AU & $\begin{array}{c}\text { B-LGBP } \\
{[\mathbf{2 7}]}\end{array}$ & $\begin{array}{c}\text { B-Geo } \\
{[\mathbf{2 7}]}\end{array}$ & $\begin{array}{c}\text { BCNN } \\
{[\mathbf{9}]}\end{array}$ & $\begin{array}{c}\text { CDPSL } \\
{[\mathbf{1}]}\end{array}$ & $\begin{array}{c}\text { DLE } \\
{[\mathbf{2 9}]}\end{array}$ & $\begin{array}{c}\text { CNN- } \\
\text { LSTM [10] }\end{array}$ & Proposed \\
\hline AU1 & 0.180 & 0.188 & $\mathbf{0 . 3 9 9}$ & 0.260 & 0.261 & 0.280 & 0.349 \\
\hline AU2 & 0.159 & 0.185 & 0.346 & 0.250 & 0.167 & 0.280 & $\mathbf{0 . 3 7 0}$ \\
\hline AU4 & 0.225 & 0.197 & 0.317 & 0.250 & 0.283 & 0.340 & $\mathbf{0 . 3 4 5}$ \\
\hline AU6 & 0.671 & 0.645 & 0.718 & 0.730 & 0.729 & 0.700 & $\mathbf{0 . 7 5 6}$ \\
\hline AU7 & 0.751 & 0.799 & 0.776 & $\mathbf{0 . 8 0 0}$ & 0.785 & 0.780 & 0.776 \\
\hline AU10 & 0.799 & 0.801 & 0.797 & $\mathbf{0 . 8 4 0}$ & 0.802 & 0.810 & 0.807 \\
\hline AU12 & 0.792 & 0.801 & 0.793 & 0.820 & 0.779 & 0.780 & $\mathbf{0 . 8 3 6}$ \\
\hline AU14 & 0.666 & 0.720 & 0.681 & 0.720 & 0.625 & $\mathbf{0 . 7 5 0}$ & 0.636 \\
\hline AU15 & 0.139 & 0.238 & 0.235 & 0.340 & $\mathbf{0 . 3 4 8}$ & 0.200 & 0.344 \\
\hline AU17 & 0.245 & 0.311 & 0.368 & 0.330 & $\mathbf{0 . 3 8 0}$ & 0.360 & 0.376 \\
\hline AU23 & 0.239 & 0.320 & 0.309 & 0.340 & $\mathbf{0 . 4 4 1}$ & 0.410 & 0.426 \\
\hline Avg & 0.442 & 0.473 & 0.522 & 0.516 & 0.508 & 0.520 & $\mathbf{0 . 5 4 7}$ \\
\hline
\end{tabular}

TABLE IV

F1-SCORE ON THE SEMAINE TESTING SET.

\begin{tabular}{|c|c|c|c|c|c|c|c|}
\hline AU & $\begin{array}{c}\text { B-LGBP } \\
{[\mathbf{2 7}]}\end{array}$ & $\begin{array}{c}\text { B-Geo } \\
{[\mathbf{2 7 ]}}\end{array}$ & $\begin{array}{c}\text { BCNN } \\
{[\mathbf{9}]}\end{array}$ & $\begin{array}{c}\text { CDPSL } \\
{[\mathbf{1}]}\end{array}$ & $\begin{array}{c}\text { DLE } \\
{[\mathbf{2 9}]}\end{array}$ & $\begin{array}{c}\text { CNN- } \\
\text { LSTM [10] }\end{array}$ & Proposed \\
\hline AU2 & 0.755 & 0.569 & 0.372 & 0.410 & 0.655 & $\mathbf{0 . 8 0 0}$ & 0.505 \\
\hline AU12 & 0.517 & 0.595 & 0.707 & 0.570 & $\mathbf{0 . 7 6 9}$ & 0.740 & 0.702 \\
\hline AU17 & 0.066 & 0.091 & 0.067 & 0.200 & 0.215 & $\mathbf{0 . 3 2 0}$ & 0.108 \\
\hline AU25 & 0.400 & 0.445 & 0.602 & 0.690 & 0.623 & $\mathbf{0 . 8 5 0}$ & 0.810 \\
\hline AU28 & 0.009 & 0.250 & 0.040 & 0.260 & 0.251 & 0.330 & $\mathbf{0 . 3 3 8}$ \\
\hline AU45 & 0.209 & 0.396 & 0.257 & 0.420 & 0.325 & $\mathbf{0 . 5 7 0}$ & 0.451 \\
\hline Avg & 0.326 & 0.391 & 0.341 & 0.425 & 0.481 & $\mathbf{0 . 6 0 0}$ & 0.486 \\
\hline
\end{tabular}

Finally, in order to compare our work with other methods in the literature, in the last experiment, a 3-fold partitioning is adopted on the combined BP4D training and validation sets, where 2 partitions are mixed with UNBC, DISFA, and SEMAINE datasets for architecture training, while the remaining partition is used for testing. We report the average F1-score of 3 runs over the 12 AUs mentioned in [32]. The obtained results were compared with other state-of-theart methods, namely JPML [31], DRML [32], CNN-RNN [5], and EAC [20], in table V. The proposed architecture outperforms the other methods on 9 AUs out of 12, and get the best average F1-score by a considerable margin.

\section{CONCLUSION}

In this work, we proposed an architecture that fuse different deep models (CNN, MLP, B-RNN) together, in order to
TABLE V

F1_SCORE ON THE 3-FOLDED BP4D DATASET.

\begin{tabular}{|c|c|c|c|c|c|}
\hline AU & JPML [31] & DRML [32] & CNN-RNN [5] & EAC [20] & Proposed \\
\hline AU1 & 0.326 & 0.364 & 0.314 & 0.390 & $\mathbf{0 . 5 6 3}$ \\
\hline AU2 & 0.256 & 0.418 & 0.311 & 0.352 & $\mathbf{0 . 4 7 1}$ \\
\hline AU4 & 0.374 & 0.430 & $\mathbf{0 . 7 1 4}$ & 0.486 & 0.570 \\
\hline AU6 & 0.423 & 0.550 & 0.633 & 0.761 & $\mathbf{0 . 7 9 1}$ \\
\hline AU7 & 0.505 & 0.670 & $\mathbf{0 . 7 7 1}$ & 0.729 & 0.768 \\
\hline AU10 & 0.722 & 0.663 & 0.450 & 0.819 & $\mathbf{0 . 8 4 3}$ \\
\hline AU12 & 0.741 & 0.658 & 0.826 & 0.862 & $\mathbf{0 . 8 7 8}$ \\
\hline AU14 & 0.657 & 0.541 & $\mathbf{0 . 7 2 9}$ & 0.588 & 0.662 \\
\hline AU15 & 0.381 & 0.332 & 0.340 & 0.375 & $\mathbf{0 . 4 3 1}$ \\
\hline AU17 & 0.400 & 0.480 & 0.539 & 0.591 & $\mathbf{0 . 6 0 2}$ \\
\hline AU23 & 0.304 & 0.317 & 0.386 & 0.359 & $\mathbf{0 . 4 3 5}$ \\
\hline AU24 & 0.423 & 0.300 & 0.370 & 0.358 & $\mathbf{0 . 5 1 2}$ \\
\hline Avg & 0.459 & 0.483 & 0.532 & 0.559 & $\mathbf{0 . 6 2 7}$ \\
\hline
\end{tabular}

capture deep appearance, geometric, and temporal features. In the core of our architecture, a new method is proposed for data balancing without adding any extra computational cost, in addition to a novel way for selecting threshold automatically based on each image. Experimental results show that the proposed technique outperforms the state-ofthe-art techniques by a significant margin.

\section{ACKNOWLEDGMENTS}

The work of Mina Bishay is a part of the NewtonMosharafa $\mathrm{PhD}$ scholarship, which is jointly funded by the Egyptian Ministry of Higher Education and the British Council.

\section{REFERENCES}

[1] T. Baltrušaitis, M. Mahmoud, and P. Robinson. Cross-dataset learning and person-specific normalisation for automatic action unit detection. In Automatic Face and Gesture Recognition (FG), 2015 11th IEEE International Conference and Workshops on, volume 6, pages 1-6. IEEE, 2015.

[2] M. S. Bartlett, G. Littlewort, I. Fasel, and J. R. Movellan. Real time face detection and facial expression recognition: Development and applications to human computer interaction. In Computer Vision and Pattern Recognition Workshop, 2003. CVPRW'03. Conference on, volume 5, pages 53-53. IEEE, 2003.

[3] J. Bergstra and Y. Bengio. Random search for hyper-parameter optimization. Journal of Machine Learning Research, 13(Feb):281305, 2012. 
[4] N. V. Chawla. Data mining for imbalanced datasets: An overview. In Data mining and knowledge discovery handbook, pages 853-867. Springer, 2005.

[5] W.-S. Chu, F. De la Torre, and J. F. Cohn. Modeling spatial and temporal cues for multi-label facial action unit detection. arXiv preprint arXiv:1608.00911, 2016.

[6] S. Dimic, C. Wildgrube, R. McCabe, I. Hassan, T. R. Barnes, and S. Priebe. Non-verbal behaviour of patients with schizophrenia in medical consultations-a comparison with depressed patients and association with symptom levels. Psychopathology, 43(4):216-222, 2010.

[7] P. Ekman and E. L. Rosenberg. What the face reveals: Basic and applied studies of spontaneous expression using the Facial Action Coding System (FACS). Oxford University Press, USA, 1997.

[8] S. Ghosh, E. Laksana, S. Scherer, and L.-P. Morency. A multi-label convolutional neural network approach to cross-domain action unit detection. In Affective Computing and Intelligent Interaction (ACII), 2015 International Conference on, pages 609-615. IEEE, 2015.

[9] A. Gudi, H. E. Tasli, T. M. den Uyl, and A. Maroulis. Deep learning based facs action unit occurrence and intensity estimation. In Automatic Face and Gesture Recognition (FG), 2015 11th IEEE International Conference and Workshops on, volume 6, pages 1-5. IEEE, 2015.

[10] S. Jaiswal and M. Valstar. Deep learning the dynamic appearance and shape of facial action units. In 2016 IEEE Winter Conference on Applications of Computer Vision (WACV), pages 1-8. IEEE, 2016.

[11] K. Jarrett, K. Kavukcuoglu, Y. Lecun, et al. What is the best multistage architecture for object recognition? In 2009 IEEE 12th International Conference on Computer Vision, pages 2146-2153. IEEE, 2009.

[12] H. Jung, S. Lee, S. Park, I. Lee, C. Ahn, and J. Kim. Deep temporal appearance-geometry network for facial expression recognition. arXiv preprint arXiv:1503.01532, 2015

[13] S. E. Kahou, X. Bouthillier, P. Lamblin, C. Gulcehre, V. Michalski, K. Konda, S. Jean, P. Froumenty, Y. Dauphin, N. BoulangerLewandowski, et al. Emonets: Multimodal deep learning approaches for emotion recognition in video. Journal on Multimodal User Interfaces, pages 1-13, 2015.

[14] S. Koelstra, M. Pantic, and I. Patras. A dynamic texture-based approach to recognition of facial actions and their temporal models. IEEE transactions on pattern analysis and machine intelligence, 32(11):1940-1954, 2010.

[15] A. Krizhevsky, I. Sutskever, and G. E. Hinton. Imagenet classification with deep convolutional neural networks. In Advances in neural information processing systems, pages 1097-1105, 2012.

[16] M. Lavelle, S. Dimic, C. Wildgrube, R. McCabe, and S. Priebe. Non-verbal communication in meetings of psychiatrists and patients with schizophrenia. Acta Psychiatrica Scandinavica, 131(3):197-205, 2015.

17] Q. V. Le, N. Jaitly, and G. E. Hinton. A simple way to initialize recurrent networks of rectified linear units. arXiv preprint arXiv:1504.00941, 2015.

18] Q. V. Le, W. Y. Zou, S. Y. Yeung, and A. Y. Ng. Learning hierarchical invariant spatio-temporal features for action recognition with independent subspace analysis. In Computer Vision and Pattern Recognition (CVPR), 2011 IEEE Conference on, pages 3361-3368. IEEE, 2011.

[19] Y. LeCun, K. Kavukcuoglu, C. Farabet, et al. Convolutional networks and applications in vision. In ISCAS, pages 253-256, 2010.

[20] W. Li, F. Abtahi, Z. Zhu, and L. Yin. Eac-net: A region-based deep enhancing and cropping approach for facial action unit detection. arXiv preprint arXiv: 1702.02925, 2017.

[21] P. Lucey, J. F. Cohn, K. M. Prkachin, P. E. Solomon, and I. Matthews Painful data: The unbc-mcmaster shoulder pain expression archive database. In Automatic Face \& Gesture Recognition and Workshops (FG 2011), 2011 IEEE International Conference on, pages 57-64. IEEE, 2011.

[22] S. M. Mavadati, M. H. Mahoor, K. Bartlett, P. Trinh, and J. F. Cohn. Disfa: A spontaneous facial action intensity database. IEEE Transactions on Affective Computing, 4(2):151-160, 2013.

[23] V. Nair and G. E. Hinton. Rectified linear units improve restricted boltzmann machines. In Proceedings of the 27th International Conference on Machine Learning (ICML-10), pages 807-814, 2010.

[24] M. Oquab, L. Bottou, I. Laptev, and J. Sivic. Learning and transferring mid-level image representations using convolutional neural networks. In Proceedings of the IEEE conference on computer vision and pattern recognition, pages 1717-1724, 2014.

[25] M. Schuster and K. K. Paliwal. Bidirectional recurrent neural net- works. IEEE Transactions on Signal Processing, 45(11):2673-2681, 1997.

[26] N. Srivastava, G. E. Hinton, A. Krizhevsky, I. Sutskever, and R. Salakhutdinov. Dropout: a simple way to prevent neural networks from overfitting. Journal of Machine Learning Research, 15(1):1929_ 1958, 2014.

[27] M. F. Valstar, T. Almaev, J. M. Girard, G. McKeown, M. Mehu, L. Yin, M. Pantic, and J. F. Cohn. Fera 2015-second facial expression recognition and analysis challenge. In Automatic Face and Gesture Recognition (FG), 2015 11th IEEE International Conference and Workshops on, volume 6, pages 1-8. IEEE, 2015.

[28] X. Xiong and F. De la Torre. Supervised descent method and its applications to face alignment. In Proceedings of the IEEE conference on computer vision and pattern recognition, pages 532-539, 2013.

[29] A. Yüce, H. Gao, and J.-P. Thiran. Discriminant multi-label manifold embedding for facial action unit detection. In Automatic Face and Gesture Recognition (FG), 2015 11th IEEE International Conference and Workshops on, volume 6, pages 1-6. IEEE, 2015.

[30] Z. Zeng, M. Pantic, G. I. Roisman, and T. S. Huang. A survey of affect recognition methods: Audio, visual, and spontaneous expressions. IEEE transactions on pattern analysis and machine intelligence, 31(1):39-58, 2009.

[31] K. Zhao, W.-S. Chu, F. De la Torre, J. F. Cohn, and H. Zhang. Joint patch and multi-label learning for facial action unit detection. In Proceedings of the IEEE Conference on Computer Vision and Pattern Recognition, pages 2207-2216, 2015.

[32] K. Zhao, W.-S. Chu, and H. Zhang. Deep region and multi-label learning for facial action unit detection. In Proceedings of the IEEE Conference on Computer Vision and Pattern Recognition, pages 33913399, 2016.

[33] X. Zhu and D. Ramanan. Face detection, pose estimation, and landmark localization in the wild. In Computer Vision and Pattern Recognition (CVPR), 2012 IEEE Conference on, pages 2879-2886. IEEE, 2012. 